\title{
DINÁMICA DE LOS NÚCLEOS AGRARIOS EN MÉXICO
}

\author{
Alida Corey Arango $\mathrm{Cruz}^{1}$ \\ Vicente Celestino Pires Silveira ${ }^{2}$
}

\section{RESUMEN}

Este artículo presenta por un lado cómo la ley e instituciones en materia agraria establecen una estructura y funcionamiento para el campo mexicano en su propiedad social, sin embargo, resalta la dinámica que ellos mismos han generado. Donde en la práctica, lo real no corresponde completamente con la legislación, siendo ésta a su vez, un producto de remanentes conceptuales los que hasta hoy, diferencian dos nociones que probablemente no tengan de fondo características suficientes para ser consideradas por la ley de formas distintas.

Palabras clave: comunidad, ejido, legislación agraria, propiedad social

\section{DINÂMICA DOS NÚCLEOS AGRÁRIOS NO MÉXICO}

\section{RESUMO}

O trabalho apresenta num primeiro plano, como a lei agraria mexicana e suas instituições, estabelecem uma estrutura e funcionamento para o campo mexicano na sua propriedade social; porém, esses núcleos agrários que conformam a propriedade social, na realidade têm sua própria dinâmica, desde a qual a prática não necessariamente está determinada pela legislação; sendo que esta, por sua vez, é um produto de reminiscências históricas que até hoje, diferenciam duas noções que provavelmente não apresentem características suficientes para ser consideradas pela lei, de formas distintas.

Palavras-chave: comunidade agrária, ejido, legislação agrária, propriedade social

\section{INTRODUCCIÓN}

Este trabajo forma parte de un estudio mayor referente a los núcleos agrarios (NA) en México, desde su formación hasta la actualidad en su dimensión histórico-legal y en la práctica. Se parte desde el conocimiento adquirido en materia legal e histórica sobre la cuestión agraria en México, para a través de fuentes de datos estadísticos e institucionales, descubrir la dinámica de ellos, cuántos NA hay en el país y su naturaleza (comunal o ejidal), cómo se distribuyen, y las relaciones que deberían presentarse según el estudio histórico y de ley anterior, donde

\footnotetext{
${ }^{1}$ Graduada en Ingeniería en Agronomía (ITVO, México). Maestra en Extensión Rural (UFSM). Doctoranda en Extensión Rural (PPGxR/UFSM). E-mail: alidacorey@gmail.com

${ }^{2}$ Graduado en Medicina Veterinaria (URCAMP). Maestro en Zootecnia (UFRGS). Ph. D. en Resource Management (University of Edimburgh). Profesor do Programa de Pós-graduação em Extensão Rural (PPGExR/UFSM). E-mail: vcpsilveira@gmail.com
} 
sobresale la cuestión de grupos indígenas (ARANGO CRUZ, 2016), que se considera importante relacionar directamente con la propiedad social ${ }^{3}$.

Actualmente la espina dorsal que ha dado forma y establecido los procedimientos en materia agraria en el país, se encuentra primeramente en la Constitución Política de los Estados Unidos Mexicanos de 1917, en el artículo 27, fracción VII

Se reconoce la personalidad jurídica de los núcleos de población ejidales y comunales y se protege su propiedad sobre la tierra, tanto para el asentamiento humano como para actividades productivas. La ley protegerá la integridad de las tierras de los grupos indígenas. (...) La ley, con respeto a la voluntad de los ejidatarios y comuneros para adoptar las condiciones que más les convengan en el aprovechamiento de sus recursos productivos, regulará el ejercicio de los derechos de los comuneros sobre la tierra y de cada ejidatario sobre su parcela. (...) La Asamblea General es el órgano supremo del núcleo de población ejidal o comunal, con la organización y funciones que la ley señale. El comisariado ejidal o de bienes comunales, electo democráticamente en los términos de la ley, es el órgano de representación del núcleo y el responsable de ejecutar las resoluciones de la asamblea (México, 5 de febrero de 1917).

Así mismo en la Ley Agraria de 1992 (LA), reglamentaria del artículo 27 Constitucional, en la que está fundamentada la normatividad referente a los predios rústicos y que incluyen a los NA que son los ejidos y comunidades agrarias; sobre éstos establece qué son, cómo están conformados, señala la operación interna en el núcleo y la de relación con el Estado que deben seguir (Cuadro 1); y decreta las estructuras institucionales agrarias.

Cuadro 1 - Tipos de la propiedad social en México.

\begin{tabular}{|c|c|c|c|}
\hline Nucleo agrario & Referencia de ley & Tipo de tierras & Órganos internos \\
\hline EJIDO & $\begin{array}{l}\text { NA propietarios de } \\
\text { las tierras que les } \\
\text { han sido dotadas o } \\
\text { las que obtuvieron } \\
\text { por otro título. }\end{array}$ & $\begin{array}{l}\text { Tierras para } \\
\text { asentamiento humano } \\
\text { Tierras para uso } \\
\text { común } \\
\text { Tierras parceladas }\end{array}$ & $\begin{array}{l}\text { Asamblea General } \\
\text { Comisariado Ejidal } \\
\text { Consejo de } \\
\text { Vigilancia }\end{array}$ \\
\hline COMUNIDAD & $\begin{array}{l}\text { NA reconocidos } \\
\text { como tal, por } \\
\text { restitución de sus } \\
\text { tierras despojadas } \\
\text { y/o que guardan el } \\
\text { estado comunal; o } \\
\text { por el procedimiento } \\
\text { de ejido a } \\
\text { comunidad }\end{array}$ & $\begin{array}{l}\text { Las que la comunidad } \\
\text { determine*, aunque la } \\
\text { ley contempla de las } \\
\text { de uso común. }\end{array}$ & $\begin{array}{l}\text { Asamblea de } \\
\text { Comuneros } \\
\text { Comisariado de } \\
\text { Bienes Comunales } \\
\text { Cualquier otra que } \\
\text { ellos elijan* }\end{array}$ \\
\hline
\end{tabular}

* Según su estatuto comunal y costumbre (art. 99, Ley Agraria)

Fuente: propia según información de la Ley Agraria

Estas instituciones son: la Secretaría de la Reforma Agraria (SRA) que se transforma en 2013 a Secretaria de Desarrollo Agrario, Territorial y Urbano

\footnotetext{
${ }^{3}$ En el estudio de Arango Cruz (2016), se aborda la terminología de tenencia de la tierra y cómo de ésta se deriva en los estudios agrarios la de propiedad social de la tierra, la que no aparece en las leyes, sin embargo, los autores a lo largo del tiempo en los estudios agrarios fueron incorporando por su carácter social (en la organización y uso colectivo de la tierra), y para diferenciarla de la propiedad privada.
} 
$\left(\right.$ SEDATU) ${ }^{4}$ que tiene como finalidad "proporcionar certeza jurídica en la tenencia de la tierra a la población, a través del impulso al ordenamiento territorial y la regularización de la propiedad rural, así como elaborar políticas públicas" (SEDATU, 2013); el Registro Agrario Nacional (RAN) para "el control de la tenencia de la tierra y la seguridad documental derivados de la aplicación de la ley" (México, 1992); la Procuraduría Agraria (PA) para "impartición de justicia agraria ,con objeto de garantizar la seguridad jurídica en la tenencia de la tierra ejidal, comunal y de la pequeña propiedad, y apoyar la asesoría legal de los campesinos" (México, 5 de febrero de 1917) y "para la defensa de los derechos de los ejidatarios, comuneros, sucesores de ejidatarios o comuneros, ejidos, comunidades, pequeños propietarios, avecindados y jornaleros agrícolas" (México, 1992); los Tribunales Agrarios compuestos por el Tribunal Superior Agrario (TSA) y los Tribunales Unitarios Agrarios, son los órganos federales a los que corresponde la impartición de justicia agraria; y el Fideicomiso Fondo Nacional de Fomento Ejidal (FIFONAFE) que es un fideicomiso público para facilitar las actividades productivas en ejidos y comunidades, administra sus fondos comunes y vigila el pago de indemnizaciones y el cumplimiento de la causa de utilidad pública en expropiaciones de bienes ejidales y comunales (SEDATU, 2013) (Figura 1).

Figura 1- Marco legal institucional agrario de México.

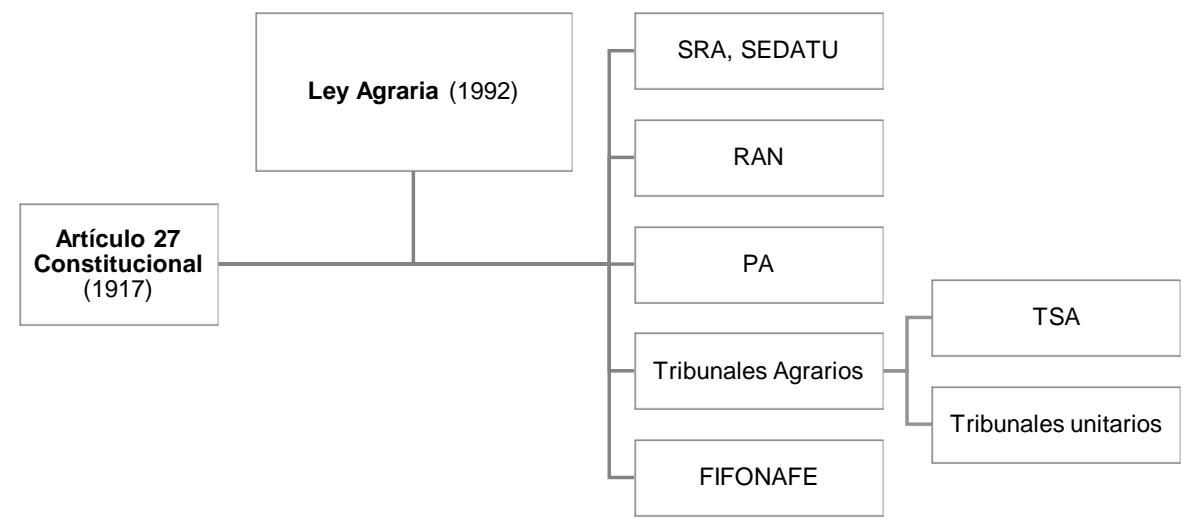

SRA: Secretaría de la Reforma Agraria, SEDATU: Secretaría de Desarrollo Agrario, Territorial y Urbano; RAN: Registro Agrario Nacional; PA: Procuraduría Agraria; FIFONAFE: Fideicomiso Fondo Nacional de Fomento Ejidal; TSA: Tribunal Superior Agrario.

Fuente: elaboración propia con información de la Ley Agraria e Instituciones Agrarias

Según Robles Berlanga (2011), de las 195 millones de hectáreas rústicas en el país, los ejidos y comunidades ocupan 105 millones en 31 mil NA, de los que 29 mil tienen documentos definidos y registrados. Este reconocimiento como NA, ya sea como ejido o comunidad inicia en 1992 con el Programa de Certificación de

\footnotetext{
4 Por decreto del 20 de mayo de 2013 en el Diario Oficial de la Federación (DOF), se aprueba el Plan Nacional de Desarrollo 2013-2018 derivándose de éste numerosos programas creados para cumplir las metas del plan, entres éstos está el Programa Sectorial de Desarrollo Agrario, Territorial y Urbano 20132018 publicado en el DOF el 16 de diciembre de 2013, en el que se establece la conclusión de la transformación de la SRA en la SEDATU, que ya se había decretado desde el 02 de enero del mismo año (DOF, 16 dic. 2013).
} 
Derechos Ejidales y Titulación de Solares Urbanos (PROCEDE), como parte de las políticas neoliberales y de reforma al artículo 27 constitucional y la ley agraria.

Así, como consecuencia de las recomendaciones del Banco Mundial para eliminar las diferencias entre propiedad privada y la ejidal así como para dar apertura al campo permitiendo la privatización de los ejidos, o la posibilidad de rentarlas, usarlas como garantía para préstamos y ya no estar obligados a trabajarlas personalmente pudiendo transmitir sus derechos parcelarios por medio de contratos comerciales 0 asociándose con más ejidatarios o inversionistas externos, y para incluir al campo mexicano en las políticas del Tratado de Libre Comercio con Estados Unidos y Canadá (MARTÍNEZ CUERO; GARCÍA RAMÍREZ, 2011).

EI PROCEDE, según la SRA en el marco del programa, el objetivo era "dar certidumbre jurídica a la tenencia de la tierra a través de la entrega de certificados parcelarios y/o certificados de derechos de uso común, o ambos según sea el caso, así como de los títulos de solares en favor de los individuos con derechos que integran los núcleos agrarios que así lo aprueben y soliciten"; después de catorce años se declara el cierre operativo del programa el 17 de noviembre de 2006 en el DOF, "en razón de que en términos de los artículos 9, 22 y 56 de la Ley Agraria, se realizó la invitación al $100 \%$ de los núcleos agrarios legalmente constituidos en el País, para su incorporación al Programa". Según las estadísticas del RAN, 92,24\% de los NA fueron certificados mediante el PROCEDE, y a causa de un porcentaje restante en 2007 se instrumenta el programa Fondo de Apoyo para Núcleos Agrarios sin Regularizar (FANAR) (PROCURADURÍA AGRARIA, 2009).

Por otro lado en un estudio realizado sobre la conformación de las nociones de ejido y comunidad, queda explícito la ligación de comunidad agraria con comunidad indígena, y aún en la legislación actual la alusión de las comunidades indígenas con la comunidad agraria es inmanente cuando se habla de restitución de las tierras a los grupos que ya las poseían desde antes de la Revolución Mexicana y que fueron enajenadas mediante la Ley de Desamortización de 1856, así como el de guardar el estado comunal ${ }^{5}$ y sus costumbres; por tanto es importante, conocer cómo es que los grupos indígenas están relacionados en los NA e intentar un acercamiento a su distribución y dinámica.

La importancia de lo anterior va más allá que de su carácter conceptual en cuanto a comunidad indígena/agraria, ya que según Robles Berlanga y Concheiro Bórquez (2004), la diversidad de México, incluye 62 lenguas vivas y más de 100 dialectos, colocándolo incluso como el tercer país con mayor diversidad étnica en el mundo.

\section{METODOLOGÍA}

De la parte documental y referente a la legislación actual, se hace un estudio en datos estadísticos e informes de las instituciones agrarias para corroborar lo concluyente a la revisión legal y de historia porque naturalmente se esperaría que en la práctica se encuentre el ejercicio de la legislación. En vista de conocer la cantidad, la distribución y la naturaleza de los NA en el país y su relación con los grupos indígenas. Para ver la relación que se establece hoy en día entre los NA, su

\footnotetext{
5 Estado comunal hace referencia a la acepción de situación, condición social y circunstancial y no al estado en su acepción de unidad política, territorio o de población. El cómo se incorporó en la ley y posteriormente a los estudios agrarios, así como su concreción en materia legal, no ha sido discutido o esclarecido por la Ley Agraria, la Constitución o los autores agrarios (ARANGO CRUZ, 2016).
} 
funcionamiento y el marco jurídico impulsado; y el cómo surge el vínculo entre comunidad agraria, comunidad indígena verificar el estudio de Arango Cruz (2016).

Se seleccionó los informes de Instituciones que trabajan directamente con la Propiedad Social de la tierra en México como el Registro Agrario Nacional, la Procuraduría Agraria; así como el Instituto Nacional de Estadística y Geografía que levanta los censos de población y vivienda ya que contiene los datos relacionados a las comunidades indígenas. Se consideró además el estudio por algunos autores que hicieron levantamientos de información geográfica, agraria y de comunidades indígenas en los que se presentan relaciones que normalmente no están disponibles en las instituciones anteriormente referidas.

\section{RESULTADOS Y DISCUSIÓN}

En el país existen 29240 ejidos y 2383 comunidades según la Procuraduría Agraria, 2011 y según datos del RAN en Reyes González et al. (2012), 29441 ejidos y 2344 comunidades que sumados representa el $53 \%$ de la superficie del país. Sin embargo según datos del RAN (2016) que muestran los datos por estado, en la suma de ejidos y comunidades totales por estado, se encuentran 29 618 ejidos y 2374 comunidades, sumando 31992 NA en el país, distribuidos por estado como se muestra en el gráfico 1.

\section{Gráfico 1- Núcleos Agrarios por estado}

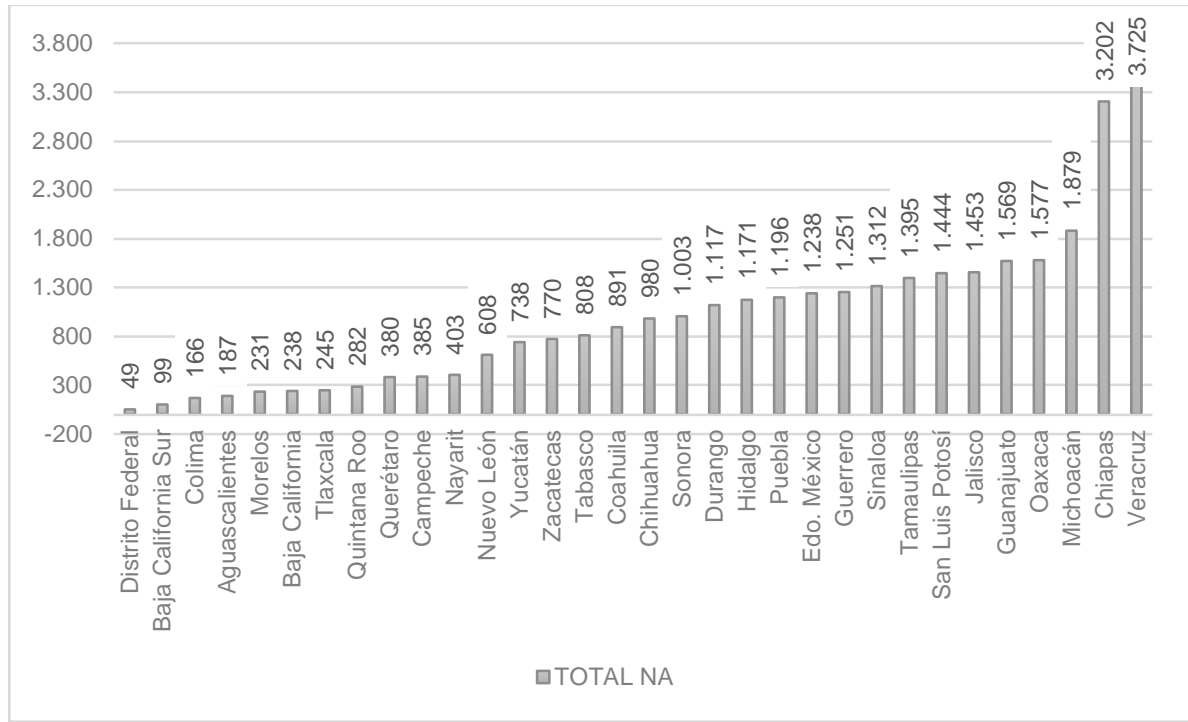

Fuente: propia con datos del RAN (2016).

La superficie de la Propiedad Social en el país comprende el 53\% del total, que representa una parte significativa del territorio, y así mismo se manifiesta por estado donde la gran mayoría de los éstos tienen importantes cantidades de ejidos y comunidades, gráfico 2, sobresale Oaxaca con el $79 \%$ de su territorio en propiedad social; el $87 \%$ de los estados tienen más del $40 \%$ de la superficie de su territorio bajo este esquema. 
Gráfico 2- Porcentajes de la superficie de propiedad social por estados

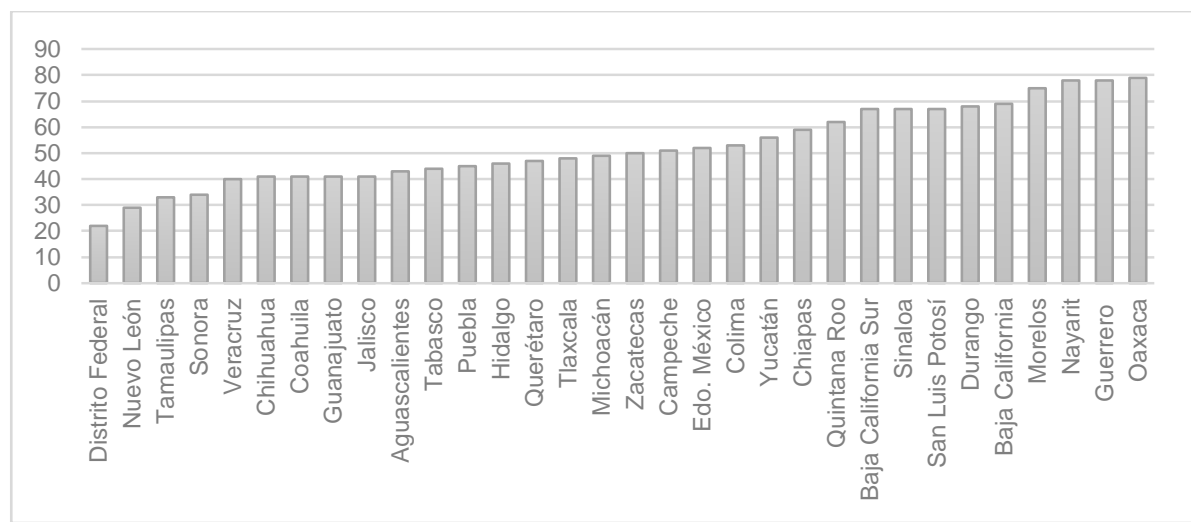

Fuente: propia con datos del RAN, 2016

A nivel nacional, la cantidad de ejidos supera por mucho a las comunidades agrarias, así mismo se ve reflejado en los datos por estado, con excepción nuevamente de Oaxaca donde el caso aparece más homogéneo, con 850 ejidos y 727 comunidades. En el caso de las comunidades Baja California Sur, Quintana Roo y Campeche, no presentan comunidades agrarias, estos dos últimos con presencia de hablantes de una lengua indígena en grado medio (10-27\% de su población de 3 y más años de edad); en tanto que los estados con mayor presencia de hablantes de una lengua indígena, en general, son los que presentan mayor número de comunidades agrarias, como Oaxaca, Guerrero, San Luis Potosí, Puebla y Chiapas (Gráficos 3 y 4).

Gráfico 3- Ejidos por estado

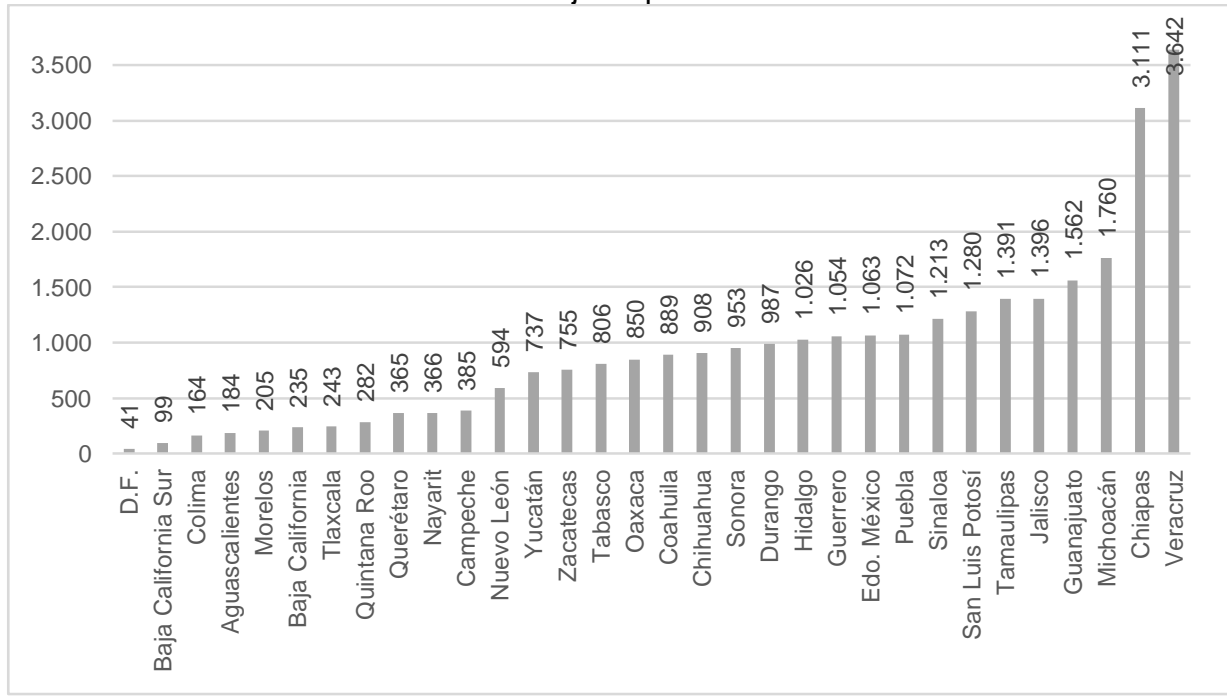

Fuente: propia con datos del RAN, 2016 
Gráfico 4- Comunidades por estado.

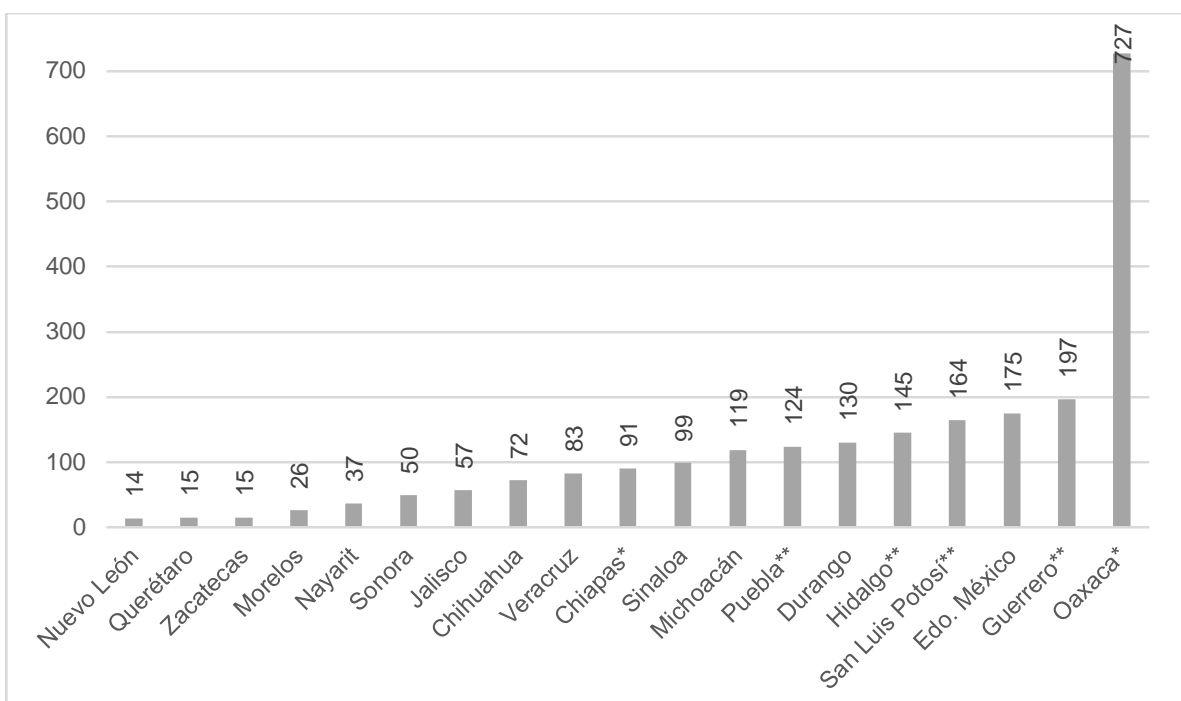

*Estados con el 27,9-32,2\% de población de 3 y más años hablante de lengua indígena; **Estados con el $10-27,8 \%$ de población de 3 y más años hablante de lengua indígena.

Fuente: propia con datos de RAN (2016) e INEGI (2015).

Según la relación que debería presentarse en cuanto a las comunidades indígenas con las comunidades agrarias, conforme se menciona en el Cuadro 1 y en el estudio de Arango Cruz (2016), se asume que por lo menos la mayoría de éstas comunidades indígenas tendría que aparecer como tierras comunales porque para eso existe la diferencia entre comunidad agraria y la de ejido, y el trato legal para cada una ha sido diferenciado sutilmente en cuanto a que las comunidades guardan un estatuto comunal y una historia de costumbre (México, 1992) y porque su posesión es "por tiempo inmemorial" (SANDOVAL AGUILAR et al., 1999, p. 91); sin embargo, es sobresaliente la diferencia entre ejidos y comunidades, donde el ejido es la figura central de la estructura agraria social del país.

No ha sido considerado por parte de los censos agrarios levantados por el RAN, o por los censos de población del Instituto Nacional de Estadística y Geografía (INEGI), la situación de los NA y las comunidades indígenas; sin embargo según datos del último censo de población y vivienda (Instituto Nacional de Estadística y Geografía (INEGI), 2010), son 16136058 indígenas $^{6}$ (gráfico 5) , de los cuáles, 9 892 193, viven en 36266 localidades de menos de 5 mil habitantes.

6 En el censo de 2010 se consideró para el conteo, la autoadscripción como indígena y no sólo mediante el criterio lingüístico que había sido la forma predominante para referirse a los grupos indígenas, esto es importante porque los datos según este criterio, rondaban entre los 8 y 10 millones de indígenas en el país y la cantidad prácticamente fue duplicada mediante la libre autodeterminación y autoadscripción en consideración de la migración de sus localidades hacia las ciudades y aún a otros países Centro de Estudios Sociales y de Opinión Pública (CESOP, 2011). 
Gráfico 5- Población indígena en México.

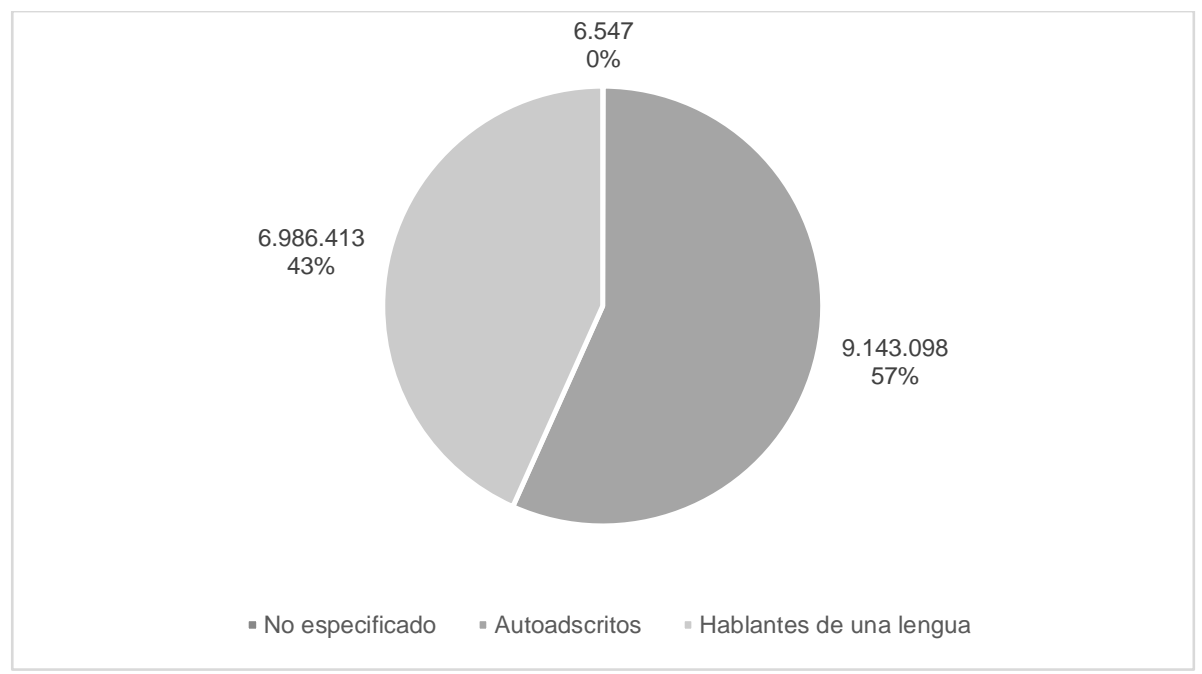

Fuente: propia con datos del INEGI (2010)

Robles Berlanga y Concheiro Bórquez (2004), realizaron un estudio complejo que involucró muchas instituciones e investigadores para determinar la cantidad de NA en México con población indígena, en esos años se contabilizaban 1 115 municipios con población indígena con 15430 NA de los cuáles se encontraron 6830 con población indígena. Estos NA, reconocen en algún grado a población indígena con derecho a la tierra; es decir, un porcentaje de su población es indígena (tabla 1), donde se destaca nuevamente la diferencia entre el número de ejidos y comunidades, en ninguno de los casos el número de comunidades supera al de los ejidos. Oaxaca es el estado con mayor número de NA con población indígena y presenta la mitad de las comunidades agrarias del país con población indígena. 
Tabla 1- NA agrarios con población indígena por estados.

\begin{tabular}{lrrr}
\hline Estado & NA & Ejidos & Comunidades \\
\hline Coahuila & 1 & 1 & 0 \\
Guanajuato & 1 & 1 & 0 \\
Jalisco & 5 & 0 & 5 \\
Tlaxcala & 5 & 5 & 0 \\
Querétaro & 7 & 6 & 1 \\
Baja California & 9 & 6 & 3 \\
Durango & 23 & 12 & 11 \\
Nayarit & 39 & 18 & 21 \\
Morelos & 49 & 42 & 7 \\
Tabasco & 106 & 105 & 1 \\
Sinaloa & 118 & 117 & 1 \\
Puebla & 153 & 127 & 26 \\
Michoacán & 155 & 93 & 62 \\
Chihuahua & 173 & 150 & 23 \\
Guerrero & 196 & 116 & 80 \\
Quintana Roo & 198 & 198 & 0 \\
Sonora & 235 & 222 & 13 \\
Campeche & 248 & 248 & 0 \\
México & 311 & 275 & 36 \\
San Luis Potosí & 451 & 301 & 150 \\
Hidalgo & 487 & 371 & 116 \\
Yucatán & 702 & 700 & 2 \\
Chiapas & 915 & 884 & 31 \\
Veracruz & 948 & 893 & 55 \\
Oaxaca & 1.295 & 671 & 624 \\
\hline Total & 6.830 & 5.562 & 1.268 \\
\hline Fuente: Robl Bea & $2904)$ & \\
\hline
\end{tabular}

Fuente: Robles Berlanga y Concheiro Bórquez (2004)

Es evidente la presencia y ligación del ejido a la estructura agraria del país, además de representar a las comunidades indígenas. Esto se debe según Robles Berlanga (2000) a que las tierras fueron reconocidas mediante restitución o dotación, en el caso de restitución de tierras, fue hasta 1940 que se estableció el procedimiento de la comunidad agraria mediante un proceso donde había que demostrar la propiedad de la tierra con los documentos del reconocimiento por parte de la Corona española en tiempos coloniales; fecha y condiciones del despojo, por lo cual muchos pueblos no podían comprobarlo y por trámites de facilitación, pasaban directo al proceso de dotación de tierras para conformar un ejido.

López-Bárcenas (2011), agrega que la restitución, al estar asociada al "estado comunal" y no con el de "pueblos indígenas" dio como resultado las diferencias actuales entre comunidad indígena y comunidad agraria puesto que una no está representada por la otra, además de existir un número importante de indígenas que son propietarios privados.

Del total de NA en el país, una gran parte está certificada (gráfico 5) por el PROCEDE y el FANAR, los pocos ejidos y comunidades no certificados al cierre operativo del PROCEDE, el (DOF, 17 nov. 2006) coloca que no ingresaron al programa "por propia voluntad o por imposibilidad derivada de problemática de índole jurídica". 
Gráfico 6- Núcleos agrarios certificados.

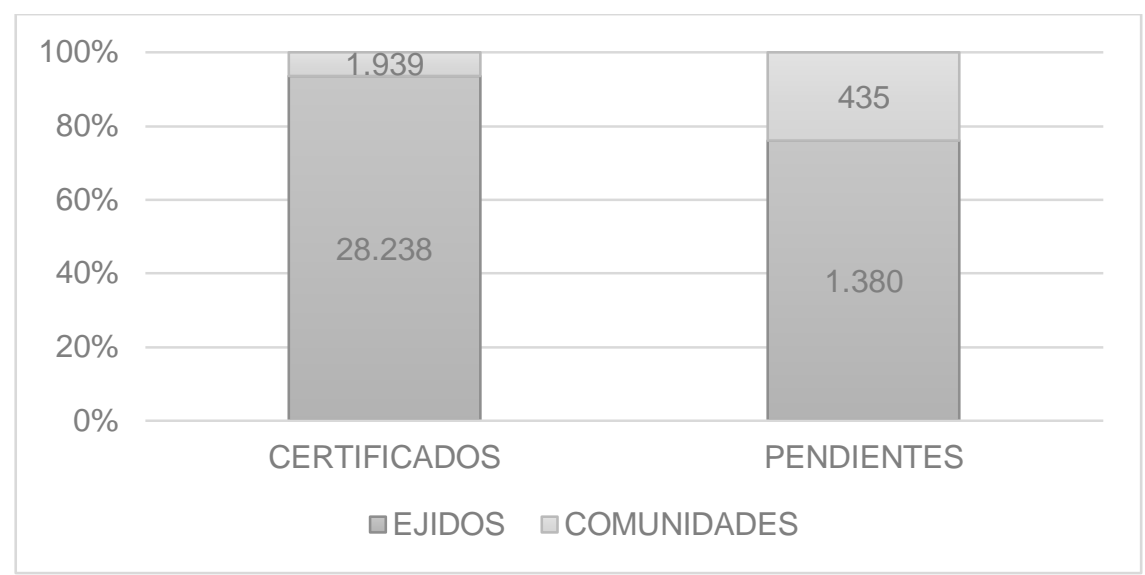

Fuente: propia con datos del Registro Agrario Nacional (2016)

En este sentido Flores Rodríguez (2008), coloca que la reforma Salinista en 1992 en materia agraria, establecía el proceso jurídico para privatizar los ejidos, y al mismo tiempo a través de diversos programas, la desincorporación del ejido para ampliar el suelo urbano en los centros de población. Trujillo Bautista (2006), agrega que el ejido en sí mismo tiene debilidad intrínseca y le atribuye parte de la crisis agraria anterior a 1992 a esta estructura. Por otro lado, los ejidos y comunidades que se resistieron al PROCEDE y al Programa de Certificación de Comunidades (PROCECOM), formaron organizaciones para pronunciarse en contra de los programas como la Red Nacional por la Defensa de la Tierra y el Territorio y en contra de PROCEDE y PROCECOM (VENTURA PATIÑO, 2008).

Por tanto, en la práctica y difiriendo de lo que podría esperarse, la dinámica de los NA está dada por la estructura de la figura 2. En la ley, se diferencian comunal y ejidal, siendo que éstos no son totalmente abordados conceptualmente para establecer las características propias de cada uno, ocasionando interminables discusiones en cuanto a la interpretación que cada institución, grupo o persona puede darle.

Figura 2- Estructura de los NA.

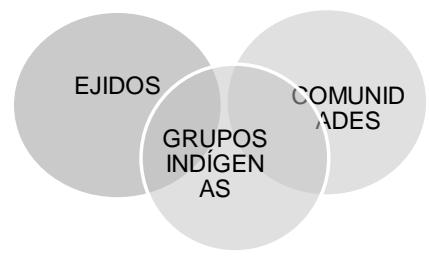

Fuente: elaboración propia

En el caso únicamente de la propiedad social, ya que como se mencionó, hay localidades indígenas con régimen de propiedad privada. El ejido es la figura 
central ya que la gran mayoría de los NA, son ejidos y son igualmente mayor el número de ejidos indígenas que las comunidades agrarias con indígenas.

\section{CONCLUSIONES}

Los NA tienen una marcada presencia a nivel nacional, ocupan un parte importante del territorio, y operan bajo un esquema reglamentario de ley. Más del $90 \%$ de los ejidos y comunidades han sido certificados y titulados, y para esto, ha sido necesario implementar de forma cabal los lineamientos que marcan la Ley Agraria y sus códigos. La misma organización interna de estos núcleos, tiene que sujetarse a la presencia de la Asamblea General, Comisariado de Bienes comunales/ejidales y el Comité de Vigilancia. Establecen además, la parcelación de tierras y la designación de las zonas de uso común. En el caso de la comunidad, la ley le da la libertad de crear otros órganos de representación interna según su estatuto comunal y de costumbre, sin embargo, las posibilidades de llevarse a cabo, quedan reducidas a las que la ley les establece.

La ambivalencia de comunidad, en el sentido agrario y de los grupos indígenas, es atribuible a la acumulación de diferentes momentos en los que la legislación no desenvolvió la noción claramente, porque la cuestión de los indígenas en México se fue desarrollando tardíamente ya que es hasta 1940 que se establecen los procedimientos en el caso de los bienes comunales y los consiguientes ajustes; y porque al parecer la prioridad al cabo de la Revolución Mexicana fue establecer ejidos, incluso la Ley Agraria en su estructura es evidente la importancia de los procedimientos en los ejidos. No se acepta la insistencia de algunos autores por colocar que la comunidad agraria y la indígena ni en ley ni en práctica se corresponden, y que esto no es sino una mala interpretación del marco legal, ya que en el estudio de la terminología legal, en retrospectiva: derechos de tierra según títulos de la Corona española que les fueron dados a los indígenas, costumbres, estado comunal, restitución de tierras a los que les fueron despojadas y posesión inmemorial, , abocan siempre a los indígenas. Esto se refuerza con el hecho de que en la ley, la tierra comunal es más protegida y difícilmente privatizable, además de flexible para dar ciertas libertades de organización interna, por tanto existe una diferenciación en el trato de los dos tipos de propiedad social, no se puede reducir como apuntan algunos, a ser indistinto el trato con comunidad y ejido.

Se puede decir que prevaleció la conformación de ejidos indígenas en lugar de las comunidades agrarias indígenas, ya que éstos son la mayoría dentro de la población rural indígena en México, y esto se debe a lo que anteriormente se mencionó sobre la requisición en la comprobación del estado comunal. Igualmente aunque en menor medida, existen comunidades indígenas en régimen de propiedad privada.

Todos los estados de México, tienen NA, y no hay una concentración de diferenciaciones que delimiten zonas o regiones características, y en todos los casos el número de comunidades es muy inferior al de los ejidos con excepción de Oaxaca. Se considera que deben haber rasgos característicos en los NA, según su tipo de conformación social, y según regiones en el país, así como diferencias entre ejido indígena, comunidad indígena y los que no lo son; pero esto requeriría más estudios a profundidad incluyendo también sus dinámicas económico-productivas.

Finalmente cabe decir que la práctica en cuanto a la naturaleza y distribución de los NA en el territorio mexicano no está apegada totalmente a la ley, sigue encontrándose una serie de diversos sentidos que continúan colocando la cuestión si aún es necesario mantener el marco legal como está o reajustarlo a la dinámica real de los NA sin violentar sus derechos e intereses de los mismos y por 
supuesto diferenciando las tierras de los grupos indígenas y su verdadera protección a su derecho sobre sus recursos que incluye por su puesto a la tierra.

\section{REFERENCIAS}

ARANGO CRUZ, A. C. Ejidos e Comunidades Agrárias em Oaxaca, México: Um estudo histórico, legal e da realidade atual. Santa Maria, Rs. Brasil, 2016. Disponible em:

<http://w3.ufsm.br/ppgexr/images/Disserta\%C3\%A7\%C3\%A3o_Alida.pdf>. Acceso en: 8 feb. 2017.

CESOP. Centro De Estudios Sociales y de Opinión Pública. Situación de los Indígenas, indicadores estadísticos: Carpeta 16, 2011.

DOF. Diario Oficial de la Federación, 16 dez. 2013. Disponible em: $<$ http://www.dof.gob.mx/nota_detalle.php?codigo=5326473\&fecha=16/12/2013>. Acceso en: 19 may. 2016.

México, 17 nov. 2006. Disponible em:

<http://dof.gob.mx/nota_detalle.php?codigo $=4937912 \&$ fecha $=17 / 11 / 2006>$. Acceso en: 23 may. 2016.

FLORES RODRíGUEZ, C. E. Suelo ejidal en México: un acercamiento al origen y destino del suelo ejidal en México. De lo comunal agrario a lo privado urbano. Cuadernos de Investigación Urbanística, n. 57, p. 21, 2008.

INEGI. Instituto Nacional de Estadística y Geografía. Censo de Población y Vivienda. México, 2010.

Encuesta Intercensal: Marco Geoestadístico Nacional, 2015.

Disponible en:

$<$ http://cuentame.inegi.org.mx/monografias/informacion/oax/poblacion/densidad.aspx ?tema=me\&e=20>. Acesso en: 7 jun. 2016.

LÓPEZ-BÁRCENAS, F. Las tierras y los territorios de los pueblos indígenas en México: Estudios de derecho constitucional local. México: Poder Judicial del Estado de Coahuila, 2011. 278-309. ISBN 6077679259. Disponible en: <http://biblio.juridicas.unam.mx/libros/libro.htm?l=3171>. Acceso en: 9 may. 2016.

MARTÍNEZ CUERO, J.; GARCÍA RAMÍREZ, R. F. (ED.). Desagrarización del campo mexicano a partir de la liberación económica. El caso de la producción de maíz en Chiapas. España, 2011. Disponible en: <http://xiiirem.ehu.es/entry/content/247/cod_048.pdf>. Acceso en: 21 may. 2016.

MÉXICO. Constitución Política de los Estados Unidos Mexicanos. 5 de febrero de 1917, 5 de febrero de 1917. Disponible en: <https://www.diputados.gob.mx\%2FLeyesBiblio\%2Fhtm\%2F1.htm\&usg=AFQjCNEpa H9iq9baJHbQO3Mzx87rZ338mA\&sig2=edhzZieEbr8oArlpdkQKfQ>. Acceso en: 2 may. 2016.

Ley Agraria. LA. 1992, 1992. Disponible en: <https://www.diputados.gob.mx\%2FLeyesBiblio\%2Fpdf\%2F13.pdf\&usg=AFQjCNH6 
W-ZYJDlkNOO5fOTruKUi9Gm6Fg\&sig2=5VjdE_FNVdmSuWKGrhSIAQ>. Acceso en: 2 may. 2016.

PROCURADURÍA AGRARIA. Estadísticas: Fondo de Apoyo para Núcleos Agrarios sin Regularizar. Estudios Agrarios, n. 42, p. 195-202, 2009. Disponible en: <http://www.pa.gob.mx/publica/rev_42/ESTADISTICAS/Estad\%C3\%ADsticas_12.pdf >. Acceso em: 21 may. 2016.

Tipología de sujetos agrarios. México, 2011.

RAN. Registro Agrario NacionaL. Núcleos Agrarios: Información Estadística. Disponível em: <http://www.ran.gob.mx/ran/index.php/informacionestadistica/nucleos-agrarios>. Acceso en: 21 may. 2016.

REYES GONZÁLEZ, J. A.; GÓMEZ BARRÓN SIERRA, J. P.; MUIS, R. O.; ZAVALA GÓMEZ DEL CAMPO, R.; RÍOS SAIS, G. A.; VILLALOBOS CAVAZOS, O. Atlas de servicios ambientales y propiedad social en México. México, 2012.

ROBLES BERLANGA, H. Ejidos y Comunidades en México: Problemas y perspectivas. Memorias del Seminario: "Propiedad Social y Servicios Ambientales", p. 30-36, 2011.

p. 123-147, 2000.

Propiedad de la tierra y población indígena. Estudios Agrarios, n. 14,

ROBLES BERLANGA, H.; CONCHEIRO BÓRQUEZ, L. Entre las fábulas y la realidad, los ejidos y comunidades con población indígena. 1. ed. Mexico: Universidad Autónoma Metropolitana; Comisión Nacional para el Desarrollo de los Pueblos Indígenas, 2004. 128 p. ISBN 970-31-0291-3.

SANDOVAL AGUILAR, Z.; ESPARZA, R.; ROJAS RABIELA, T.; OLMEDO, R. Guía de restitución y dotación de tierras y de reconocimiento, confirmación y titulación de bienes comunales del Archivo General Agrario. México: CIESAS, 1999. (Colección Agraria). ISBN 968-496-376-9.

SEDATU. Guía de Derechos Individuales y colectivos de los sujetos agrarios, 2013.

TRUJILLO BAUTISTA, J. M.. El sistema ejidal, inhibidor del desarrollo rural: Casos de los municipios de Casas y de Güemez, Estado de Tamaulipas, México, 2006.

VENTURA PATIÑO, M. D. C. Nueva Reforma Agraria neoliberal y multiculturalismo. Territorios indígenas, un derecho vuelto a negar. Revista Pueblos y fronteras, n. 5, 2008. Disponible en:

<http://www.pueblosyfronteras.unam.mx/a08n5/art_06.html>. Acceso en: 24 may. 2016. 2008. 